\title{
Raloxifene but not alendronate can compensate the impaired osseointegration in osteoporotic rats
}

\author{
Leonardo Perez Faverani ${ }^{1}$ - Tárik Ocon Braga Polo ${ }^{1}$ - Gabriel Ramalho-Ferreira ${ }^{1}$. \\ Gustavo Antonio Correa Momesso ${ }^{1}$. Jaqueline Suemi Hassumi ${ }^{1,2}$ - Ana Cláudia Rossi ${ }^{3}$. \\ Alexandre Rodrigues Freire ${ }^{3}$ - Felippe Bevilacqua Prado $^{3}$ • Eloá Rodrigues Luvizuto ${ }^{4}$. \\ Reinhard Gruber $^{5} \cdot$ Roberta Okamoto ${ }^{2}$
}

Received: 11 July 2016 / Accepted: 16 March 2017 /Published online: 29 March 2017

(C) Springer-Verlag Berlin Heidelberg 2017

\begin{abstract}
Objectives Alendronate and raloxifene, a bisphosphonate and a selective estrogen modulator, respectively, are established osteoporosis therapies. Current evidence suggests that simultaneous application of osteoporosis therapies modulates osseointegration. However, alendronate shows inconsistent findings and raloxifene has not been studied comprehensively. This study aimed to evaluate the bone dynamics and molecular and microstructural features at the peri-implant bone interface in osteoporotic rats.

Materials and methods Thirty female rats underwent ovariectomy and were fed a diet low in calcium and phosphate and treated with alendronate or raloxifene for 30 days or underwent fictional ovariectomy surgery (SHAM) prior to implant insertion in the tibia; osteoporosis therapies continued thereafter. After 42 days, peri-implant bone was evaluated by histometric and micro-CT analysis. Fluorochrome incorporation and gene expression was determined to evaluate bone turnover.
\end{abstract}

Leonardo Perez Faverani

leobucomaxilo@gmail.com

1 Department of Surgery and Integrated Clinic, Division of Oral and Maxillofacial Surgery, São Paulo State University (UNESP), School of Dentistry, Araçatuba, São Paulo 16015-050, Brazil

2 Department of Basic Sciences, São Paulo State University (UNESP), School of Dentistry, Araçatuba, São Paulo 16015-050, Brazil

3 Department of Morphology, Piracicaba Dental School, University of Campinas (UNICAMP), Piracicaba, SP, Brazil

4 Department of Surgery and Integrated Clinic, Division of Integrated Clinic, São Paulo State University UNESP), School of Dentistry, Araçatuba, São Paulo 16015-050, Brazil

5 Department of Oral Biology, Medical University of Vienna, Wien, Austria
Results We report here that alendronate had no impact on bone-to-implant contacts and the mineral apposition rate. The RANKL/OPG ratio and local bone volume, however, were increased compared to the untreated osteoporotic rats. Even though signaling to bone resorption activity through RANKL production was observed in the alendronate group, the blockade of bone resorption activity that occurs in decorrence to alendronate activity took place and resulted in an increase in bone volume. Raloxifene significantly increased osseointegration in osteoporotic rats, as indicated by bone-to-implant contacts, mineral apposition, and local bone volume. Raloxifene, however, had no considerable impact on the RANKL/ OPG ratio compared to untreated osteoporotic rats. As expected, the SH group showed higher bone-to-implant contacts and mineral apposition rates than the untreated osteoporotic rats.

Conclusions These findings suggest that raloxifene but not alendronate can compensate for the impaired osseointegration in osteoporotic rats.

Clinical relevance Regarding the superiority of raloxifene observed in the improvement of bone dynamics response, this statement suggests that raloxifene could be a good option for osteoporosis patients in oral rehabilitation procedures.

Keywords Osteoporosis · Dental implants $\cdot$ Alendronate · Raloxifene $\cdot$ Microscopy

\section{Introduction}

Osteoporosis is characterized by decreased bone mass and a deterioration of bone microstructure that frequently occurs in elderly population, particular in post-menopausal women $[1,2]$. The elderly population also has a growing demand for dental care 
including implant therapy. However, retrospective studies and prospective clinical trials have indicated a lower success rate of dental implants in patients with osteoporosis compared to a respective control population [2-5]. The studies are heterogeneous in design; thus, the impact of osteoporosis and of osteoporosis therapy in particular on the osseointegration of dental implants has not been fully investigated.

Among the most widely used osteoporosis therapies are orally administered bisphosphonates, including sodium alendronate [2, $6,7]$. Upon entering circulation, alendronate rapidly adsorbs to sites of bone turnover and is exclusively released by the boneresorbing osteoclasts, which are also the target cells [8]. Alendronate blocks intracellular enzymes that are required for the mevalonate pathway, thereby affecting cytoskeletal reorganization and vesicular fusion. Consequently, bisphosphonates control osteoclast activation and survival, thereby inhibiting the resorptive activity of osteoclasts $[8,9]$. Another class of drugs consists of selective estrogen receptor modulators. Raloxifene hydrochloride is the only drug of this class approved for the treatment of osteoporosis [5, 10-12]. Both therapies reduce the fracture risk in the lumbar spine; the impact on osseointegration however is beginning to be discovered [10] .

Systemic treatments with raloxifene showed an increase of removal torque and the bone-to-implant contacts of miniscrews placed in the tibia of osteoporotic rats $[5,10]$. There was also a higher removal torque by alendronate in OX rat tibias [13]. Alendronate could even double the boneto-methyl methacrylate cement rods in the tibia of OX rats [14]. Another bisphosphonate, zoledronic acid, supported osseointegration parameters in OX $[15,16]$ but not in normal rat tibia implantation models [17]. As summarized in a recent review, 12 animal studies showed that systemic bisphosphonates significantly increased bone volume and bone-to-implant contact under osteoporotic conditions. Two studies, however, reported no significant difference in osseointegration among OX animals [18]. Thus, there are limited data on the impact of raloxifene on osseointegration, and the reports on bisphosphonates on osseointegration are not completely proven.

Therefore, the aim of this study was to assess the periimplant bone dynamics in osteoporotic rats treated with sodium alendronate or raloxifene that underwent ovariectomy (OX), compared with SHAM (SH) rats without treatment.

\section{Material and methods}

\section{Osteoporosis induction and pharmacologic treatment}

Thirty female rats (Rattus norvegicus, albinus, Wistar; $n=10$ ) weighting about $250 \mathrm{~g}$ were provided from the Central Vivarium of the Aracatuba Dental School UNESP. The Ethics Committee in the Use of Animals (CEUA-2012-
01096) approved this study. Rats were anesthetized with a combination of $5 \mathrm{mg} / \mathrm{kg}$ xylazine (Coopazine; Coopers Brasil Ltda, Campinas, São Paulo, Brazil) and $50 \mathrm{mg} / \mathrm{kg}$ of ketamine hydrochloride (Vetaset; Fort Dodge Saúde Animal Ltda, Campinas, São Paulo, Brazil). Bilateral incisions were performed to remove the ovaries $(\mathrm{OX})$ and for sham operations, in which ovaries were only exposed without ablation SHAM (SH). Rats were kept in individual cages to daily evaluate the estrous cycle [19]. Osteoporosis was induced by combining bilateral ovariectomy with a calcium and phosphatepoor diet [20]. The SH group received a balanced diet (Nuvilab, Curitiba, PR, Brazil) containing 1.4\% calcium and $0.8 \%$ phosphate, with water ad libitum. The OX group received a diet containing $0.1 \%$ calcium and $0.5 \%$ phosphate (Rhoster Ind. Com., Vargem Grande Paulista, SP, Brazil) and water ad libitum $[10,21]$. Treatment with sodium alendronate (AL; $0.1 \mathrm{mg} / \mathrm{kg} /$ day) [22] or raloxifene (RL; $1 \mathrm{mg} / \mathrm{kg} /$ day) $[23,24]$ started 8 days after ovariectomy. Drugs were administrated until euthanasia.

The doses of $\mathrm{AL}$ and $\mathrm{RL}$ were chosen according to the correlation between the doses for treating osteoporosis in humans and animal weight. Moreover, previous studies already standardized the same doses in osteopenic rats [16-18].

\section{Implant placement}

Animals underwent $8 \mathrm{~h}$ fasting prior to the administration of local and systemic anesthesia with $50 \mathrm{mg} / \mathrm{kg}$ of ketamine intramuscularly and $5 \mathrm{mg} / \mathrm{kg}$ xylazine (mepivacaine; $0.3 \mathrm{ml} / \mathrm{kg} \mathrm{2 \%}$, adrenaline 1:100,000, Septodont, SaintMaur-des Fossés, France). The medial portion of the tibia was disinfected with povidone-iodine and exposed by a 2 $\mathrm{cm}$ incision. A 1.4-mm-diameter spiral bur mounted on an electric motor (BLM 600®; Driller, São Paulo, SP, Brazil) at a speed of $1000 \mathrm{rpm}$ under irrigation with isotonic $0.9 \%$ sodium chloride (Fisiológico ${ }^{\circledR}$, Laboratórios Biosintética Ltda ${ }^{\circledR}$, Ribeirão Preto, SP, Brazil) was used. Implants were placed manually with a square digital wrench. Grade 4 titanium implants, $2 \mathrm{~mm}$ in diameter and $4 \mathrm{~mm}$ in length with acid-etched surfaces, were implanted bilaterally in each tibia (Implalife Biotechnology, Jales, São Paulo, Brazil). Deeper layers were sutured with a resorbable thread (Poliglactina 910, Vicryl, Ethicon, Johnson \& Johnson Prod, São José dos Campos, Brazil), and the external layer was sutured with a monofilament thread (Nylon 5.0, Ethicon, Johnson \& Johnson Prod, São José dos Campos, Brazil). In the immediate post-operative period, animals received a single injection of intramuscular pentabiotic $(0.1 \mathrm{ml} / \mathrm{kg}$; Fort Dodge Saúde Animal Ltda, Campinas, São Paulo, Brazil) and sodic dipyrone $(1 \mathrm{mg} / \mathrm{kg} / 1$ day; Ariston, Indústrias Químicas e Farmacêuticas Ltda, São Paulo, Brazil) [10]. 


\section{Fluorochrome administration}

For fluorochrome staining, $20 \mathrm{mg} / \mathrm{kg}$ of calcein and $20 \mathrm{mg} / \mathrm{kg}$ of alizarin were administered at 14 and 42 days, respectively, after implant placement [25]. At 60 days, euthanasia was performed with a lethal dose of anesthetic (thiopental $100 \mathrm{mg} / \mathrm{kg}$, Cristalia Ltda, Itapira, SP, Brazil).

\section{Micro-CT analysis}

Computed micro tomography was performed with a SkyScan 1176 (Bruker microCT, Aartselaar, Belgium). The region of interest (ROI) was a rectangular area, $0.5 \mathrm{~mm}$ high and $0.8 \mathrm{~mm}$ wide, corresponding to the valleys between the third and fifth screws of the implant. Analysis was based on 100 slices $(874.3 \mu \mathrm{m})$ and was performed with the CT Analyzer (SkyScan, Leuven, Belgium; Fig. 1). Images were converted to a gray scale using an interval of values in the range from 0 to 255 , with the minimum value equal to 70 and the maximum value equal to 100 in all of the groups. These values were determined on the basis of the visualization of the cancellous bone structure of the ROI (Fig. 1). The volumetric parameters were elected according to guidelines from the American Society of Bone and Mineral Research (ASBMR) [26]. Therefore, the percentage of bone volume (BV/TV), trabecular thickness (Tb.Th), trabecular separation (Tb.S), and trabecular number (Tb.N) were obtained.

\section{Processing of specimens for histology and histometric analysis}

After micro-CT analysis, the tibiae were fixed in paraformaldehyde for $48 \mathrm{~h}$, washed in running water for $24 \mathrm{~h}$, and dehydrated in ascending concentrations of alcohol. Embedding in light-curing resin (Technovit 7200 VLC, EXAKT Advanced Technologies, Norderstedt, Germany) was followed by processing with the EXAKT Cutting and Grinding equipment to reach an average thickness of $80 \mu \mathrm{m}$, and these slices were adapted on the histological blade surface.

\section{Confocal microscopy}

A longitudinal section was obtained at the region corresponding to the third, fourth, and fifth turns of the implants. Images were taken by a Leica CTR 4000 CS SPE laser confocal microscope (Leica Microsystems, Heidelberg, Germany) using a $10 \times$ objective (original zoom 100).

The images obtained by confocal microscopy were imported using image analysis software (ImageJ, Processing Software and Image Analysis, Ontario, ON, Canada) to calculate the fluorochrome area in the region of the implant in square micrometers. Firstly, using the "color threshold" tool, the images of each experimental group (OX, SH, RL, and AL) were standardized in hue, saturation, and brightness, so that the fluorochromes were highlighted. The green fluorescent color (calcein) was selected, and the "measure" tool was used

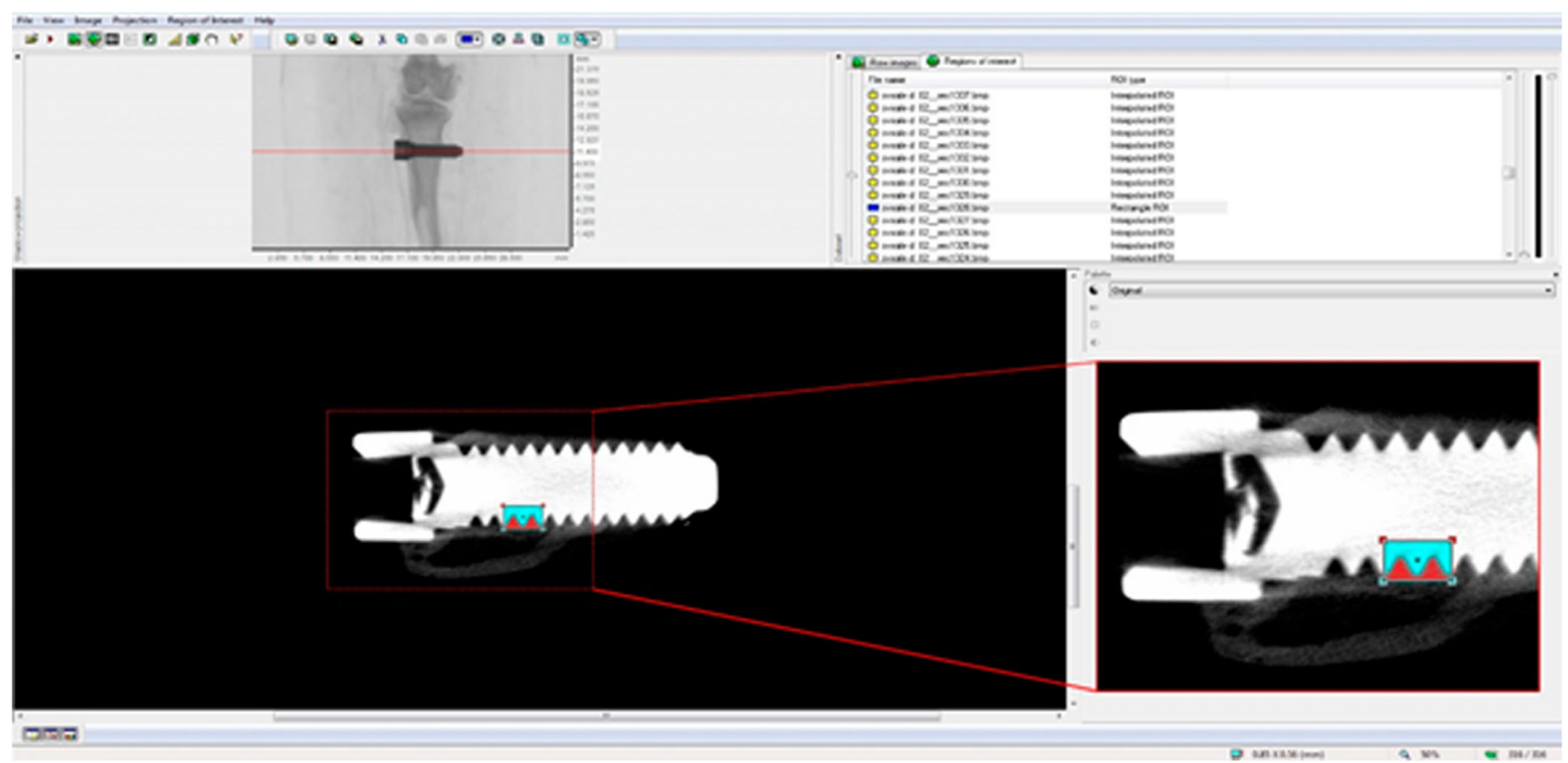

Fig. 1 ROI demarcation in the CT Analyzer software. The red frame shows an approach for better viewing of the rectangular area corresponding to the valleys between the third and fifth turns of the implant 
to calculate the corresponding area in square micrometers. Subsequently, fluorescent red (alizarin) was measured, thus making it possible to interpret the peri-implant bone dynamics in each condition tested. For all images, the fourth implant thread was standardized (middle implant thread) to calculate the area marked by the fluorochromes.

Using the same slides, the mineral apposition rate (MAR) was measured according to the American Society of Bone and Mineral Research (ASBMR) in 2013 [26]. The images were analyzed on the ImageJ software (Software for Image Processing and Analysis, Ontario, ON, Canada) using a "straight" toll to calculate the distance between the external surfaces of each precipitate fluorochrome (beginning of calcein precipitation up to the end of alizarin precipitation) in two turns of the implants (fourth and fifth). These values were divided by 28 to represent the interval days between both injections. The result represented the value of mineral apposition by day (MAR) in micrometers.

After that, the same slices were washed and stained with Alizarin Red and Stevenel's Blue. The slides were photomicrographs in optic microscopy (Carl Zeiss, Oberkochen, Germany), and the images were measured in the ImageJ software (Software for Image Processing and Analysis, Ontario, ON, Canada) using a straight toll to calculate the perimeter between the fourth and fifth turns of the implants on the boneimplant interface (bone implant contact, BIC) in micrometers. Besides that, the newly formed bone (NBF) was measured through a "free hands" toll to calculate the bone area formed at the middle implant thread (fourth turn of the implants) in square micrometers.

The histological sections were analyzed after completion of the study without knowledge of animal number or group. All parameters comply with the nomenclature and were calculated according to the histometry nomenclature committee of the ASBMR [26].

\section{Processing of specimens for immunohistochemistry}

After euthanasia, the tibiae were removed and immediately fixed in a $10 \%$ buffered formalin solution (Reagentes Analíticos, Dinâmica Odonto Hospitalar Ltda, Catanduva, SP, Brazil) for $48 \mathrm{~h}$ and soaked in running water for $24 \mathrm{~h}$. The tissue was decalcified in 10\% EDTA solution for 6 weeks and then dehydrated in an increasingly concentrated alcohol solution sequence. Diaphonization was performed with xylene, and finally, the specimens were embedded in paraffin. The tissue block was sectioned at a thickness of $5 \mu \mathrm{m}$ using a microtome, and the sections were mounted on slides.

Immunostaining was visualized using the indirect immunoperoxidase detection method. Blocking of nonspecific reactions was performed by inactivation of endogenous peroxidase, using 3\% hydrogen peroxide (Merck, Sao Paulo, SP, Brazil), $1 \%$ bovine serum albumin (Sigma-Aldrich Ltda.,
Sao Paulo, SP, Brazil), and 20\% nonfat powdered milk. Antigen retrieval was achieved with a citrate phosphate buffer ( $\mathrm{pH}$ 6.0) in the presence of moist heat.

The primary antibodies used were against tartrate-resistant acid phosphatase (TRAP), osteoprotegerin (OPG), receptor activator of nuclear factor kappa-B ligand (RANKL), and osteocalcin (Santa Cruz Biotechnology, Dallas, TX, USA). These proteins were chosen in order to analyze the cellular responses to bone resorption (TRAP), remodeling (OPG and RANKL), and bone mineralization (osteocalcin). The secondary antibody used was a biotinylated anti-goat antibody produced in rabbits (Pierce Biotechnology, Rockford, IL USA), with biotin and streptavidin (Dako, Glostrup, Denmark), and diaminobenzidine (Dako, Glostrup, Denmark) as the chromogen. Counter-staining was performed with Harris hematoxylin.

The staining was evaluated by the attribution of "scores" that were assigned according to the presence of immunostained cells in the region of the peri-implant bone repair 20 . Analysis was performed by light microscopy (Leica DMLB, Heerbrugg, Switzerland), and scores were assigned to represent (1) light staining, (2) moderate staining, (3) or intense staining. A higher score reflected an increase in the number of cells positively stained by diaminobenzidine in the area. The raters' scores were subjected to the Kappa test where the index was set to $>0.8$, indicating that the observed scores were consistent. Substituting the primary antibody with serum from the primary antibody host species served as the negative control. The immunohistochemical reactions were performed at 42 days after implant placement.

\section{Molecular analysis (RT-PCR)}

Implants were removed through reverse torque, and the periimplant tissue was collected, washed in phosphate-buffered saline, and stored in liquid nitrogen. Total RNA was extracted with TRIzol reagent (Promega Corporation, Madison, WI, USA) and converted into cDNA (Life kit; Life Technologies, Invitrogen, Carlsbad, CA, USA). Real-time PCR was performed with the StepOnePlus (Applied Biosystems, Waltham, MA USA) using SYBR Green (Applied Biosystems). Beta-actin and beta-2 microglobulin (Life Biotechnologies, Invitrogen, Carlsbad, CA, USA) were used for normalization of OPG and RANKL expression using the delta CT method. Primer sequences have been reported recently [27]. Data represent quadruplicates.

\section{Statistical analysis}

The fluorochrome area (calcein/alizarin), MAR values, BIC, $\mathrm{NB}$, and PCR reactions (OPG and RANKL) in the periimplant region were compared statistically using SigmaPlot 12.3 Exact Graphs and Data Analysis program (San Jose, 
CA, USA). Two-way ANOVA (osteoporosis group vs. fluorochromes) and one-way ANOVA (osteoporosis groups) were applied, and for the interactions with statistical significance, Tukey (fluorochromes) and Holm-Sídak (BIC and NB) posttests were performed. Two-way ANOVA tests and Tukey post-tests were applied for immunohistochemical scores in the comparisons for OPG, RANKL, and OC (osteoporosis groups vs. periods). For all tests, the significance level used was $5 \%$.

\section{Results}

\section{Histology and histometric analysis}

The histological picture shows mature lamellar bone that files the treats of the implants with no signs of inflammation. Also visible in all groups was the bone formed around the screw and a great quantity of medullar bone, once the evaluated area was restricted to the third to fifth screws inserted in the medullar area. OX alone or the combination with alendronate and raloxifene failed to cause any visible changes on bone morphology (Fig. 2). Histomorphometric data indicated a higher bone-to-implant contact with raloxifene but not with alendronate compared to OX-alone controls (Fig. 3a). OX caused the expected decrease in bone-to-implant contacts compared to $\mathrm{SH}$ controls. In agreement, new bone formation was not modulated by alendronate but was considerably increased by raloxifene to the levels of the SH controls (Fig. 3b).

\section{Fluorochrome precipitation}

This study revealed that, in line with the histometric data, alizarin precipitation is somewhat lower in OX and its combination with alendronate than the raloxifene group and the $\mathrm{SH}$ controls (Fig. 4). Interestingly, calcein precipitation showed the reciprocal picture, being higher in OX and alendronate than the raloxifene group and the SH controls (Fig. 4). Thus, alendronate failed to support osseointegration, while raloxifen had an anabolic activity on osseointegration parameters in this OX model. In agreement with this observation, it is important to note that the mineral apposition rate (MAR) was increased in the SH group, followed by raloxifene. Both of them were superior to the OX and alendronate groups (Fig. 5).

The representation of peri-implant bone turnover can be seen in Fig. $6 \mathrm{a}-\mathrm{d}$. The precipitation of calcium in the organic matrix indicated fluorescence staining, as indicated by calcein (green color) and alizarin (red staining). The green fluorescent lines indicate old bone, while the red lines indicate renewal bone. The quantification of the area of calcein/alizarin staining (old bone/new bone) through two-way ANOVA analysis showed that all interactions between the osteoporosis vs. fluorochromes groups were statistically significant $(P<0.05)$. The intragroup analysis showed a statistically significant difference between the expression of calcein and alizarin $(P<0.001)$, always with lower values for calcein and higher values for alizarin red (Fig. 4).

The highest rate of peri-implant bone remodeling was observed in the SH and RL groups, which showed alizarin precipitation to be higher than calcein. These groups showed no statistical differences for both calcein $(P=0.376)$ and alizarin $(P=0.728)$. The interactions between the $\mathrm{OX}$ and $\mathrm{AL}$ groups ox

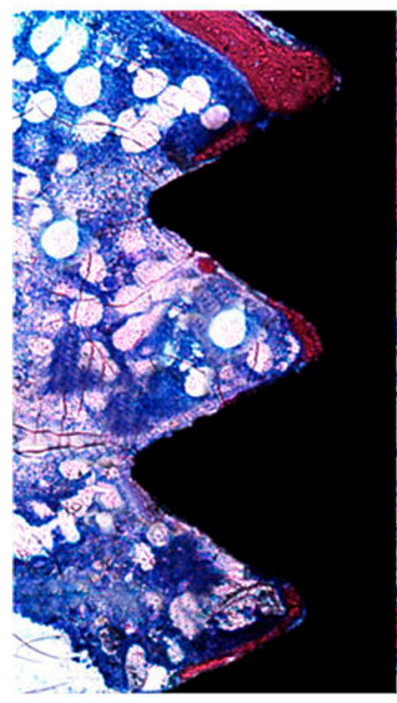

SH

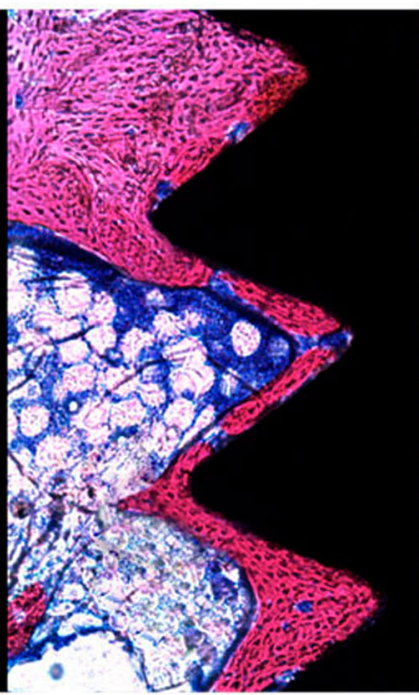

AL

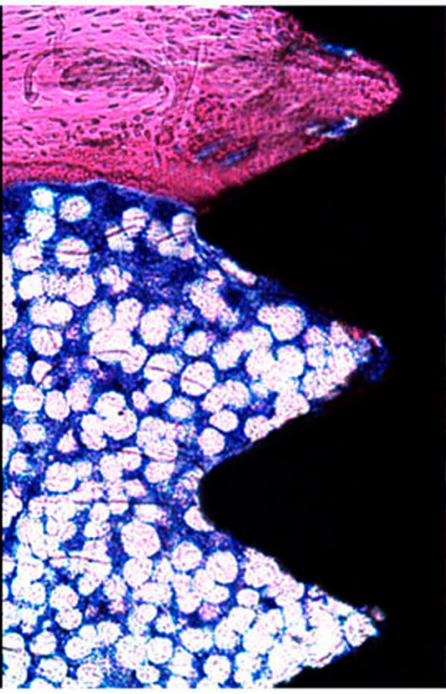

RL

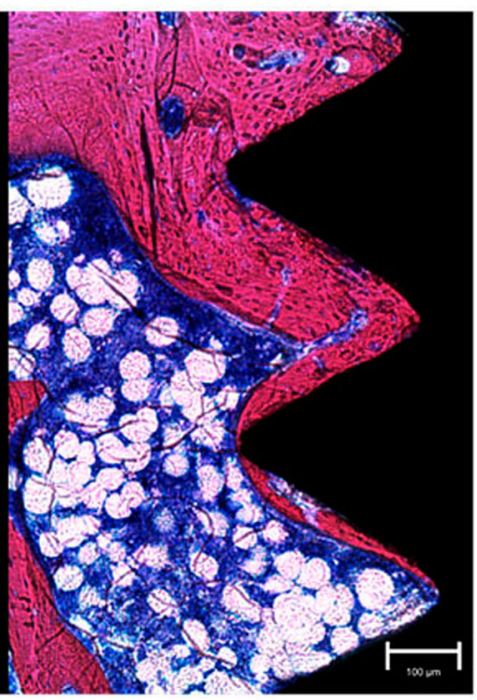

Fig. 2 Fluorescent staining in the calcium matrix by calcein (green) and alizarin (red), showing the dynamics of the peri-implant bone. Experimental groups: a $\mathrm{SH}$; b OX; c $\mathrm{AL}$; and $\mathbf{d} \mathrm{RL}$ 


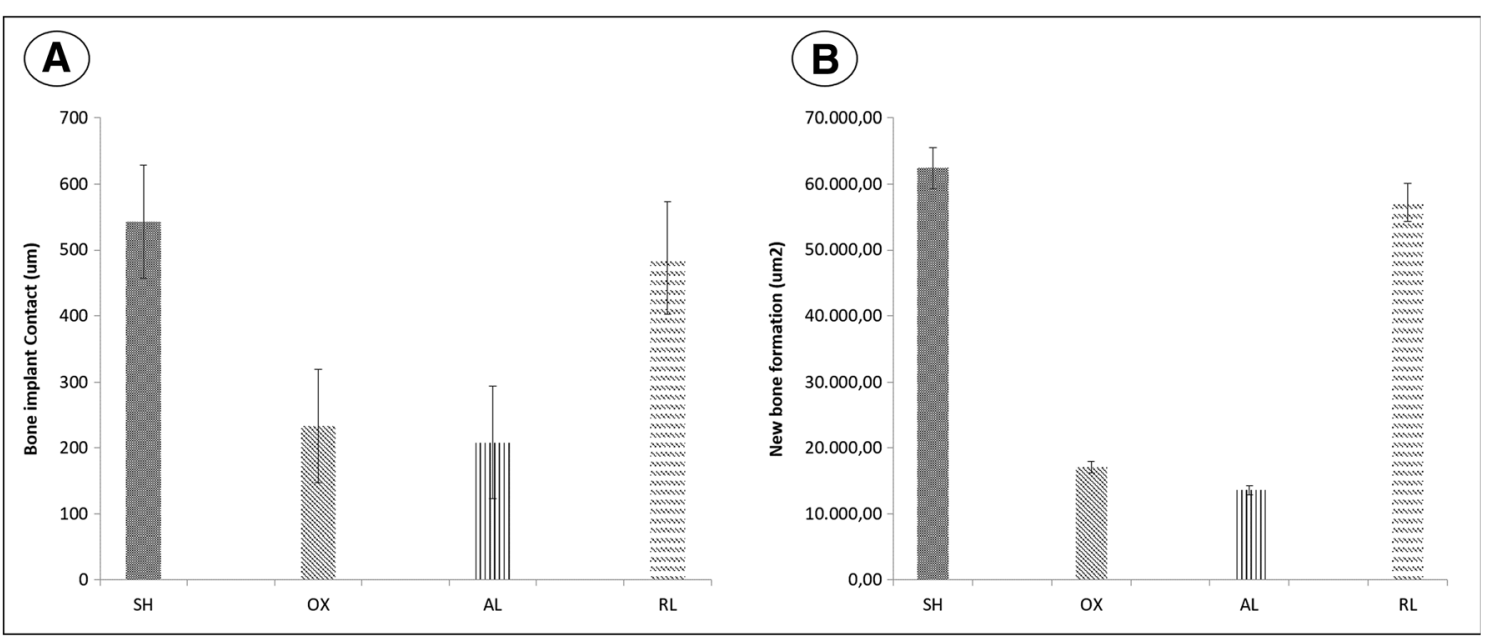

Fig. 3 Histological images on the bone/implant interface from experimental groups (OX, $\mathrm{SH}, \mathrm{AL}$, and $\mathrm{RL})$ at 42 days. Note the bone formed close to the turns of the implants, especially in the SH and RL groups.

Also, the considerable quantity of bone marrow in the OX and AL groups shows delay of bone repair. (Stevenel's blue and Alizarin red staining; $\times 20$ original magnification).

were also not significant (calcein $P=0.445$; alizarin $P=0.673$ ) because they had the lowest bone turnover rates. The other comparisons (OX vs. RL, RL vs. AL, SH vs. OX, and SH vs. AL) were statistically significant $(P<0.05$; Fig. 4).

The MAR showed greater values in the RL groups and lower values in the OX groups. The comparisons between the groups (OX vs. RL, OX vs. SH, AL vs. RL, and $\mathrm{SH}$ vs. AL) presented statistically significant differences $(P=0.001$, HolmSídak test). The other interactions (OX vs. AL and RL vs. SH) showed similar results $(P>0.05$, Holm-Sídak test; Fig. 5).

\section{Immunostaining results}

The presence of osteoprotegerin (OPG; Fig. 7) and RANKL (Fig. 8) was evaluated in order to characterize the rate of bone remodeling. Osteocalcin characterized the mineralization rate and maturity of bone tissue formed, and TRAP, measured after

42 days, indicated the presence of resorption activity of osteoclasts in the bone tissue examined.

The proteins OPG and RANKL are part of the tumor necrosis factor family and participate in the signaling of cellular responses involved in bone remodeling. The expression of OPG compared with RANKL may show a tissue response tending to bone formation (predominance of $\mathrm{OPG}$ ), bone resorption (predominance of RANKL), or balanced bone-remodeling rates (equal levels of OPG and RANKL).

In the two experimental groups, we observed that a positive OPG score was mild (1) at 42 days in the SH group. In the same group, RANKL staining was moderate to intense (2-3) at 42 days. In the AL and RL groups, both RANKL and OPG exhibited a moderate score (2). The presence of OPG and RANKL indicated positively stained osteoblastic lineage cells.

Fig. 4 Histometric data measured through bone implant contact (BIC) (a) and new bone formation area (NB) (b) on the bone/implant interface from experimental groups $(\mathrm{OX}, \mathrm{SH}, \mathrm{AL}$, and RL) at 42 days. *, \# denotes statistically significance difference $(P<0.05)$

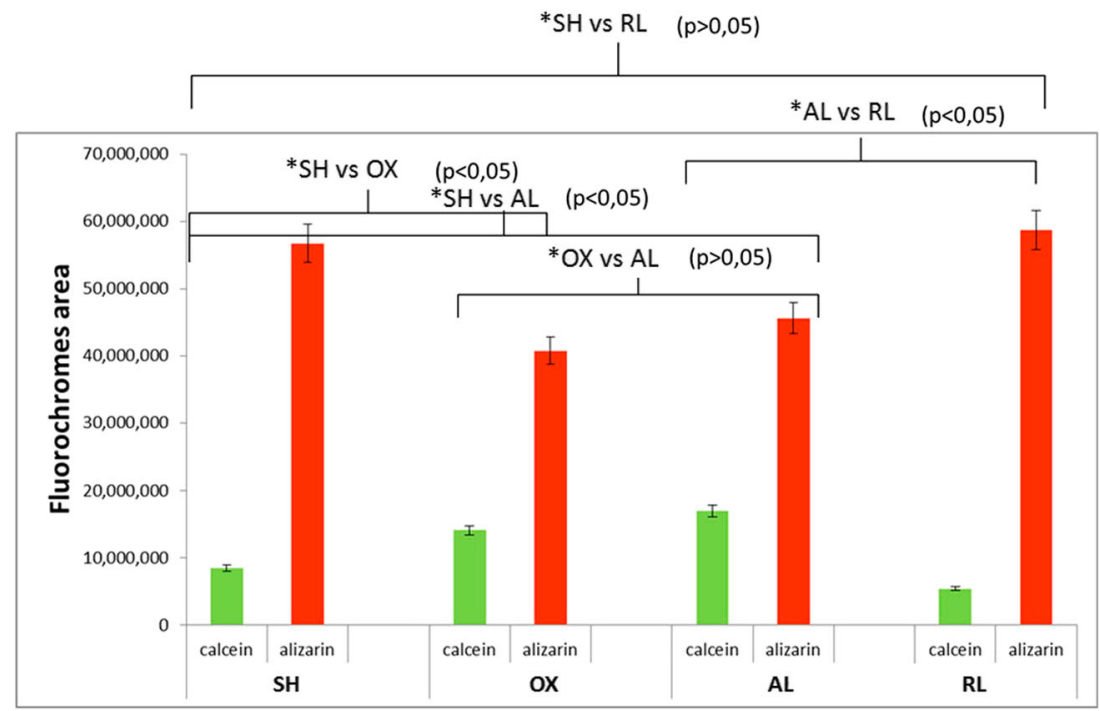




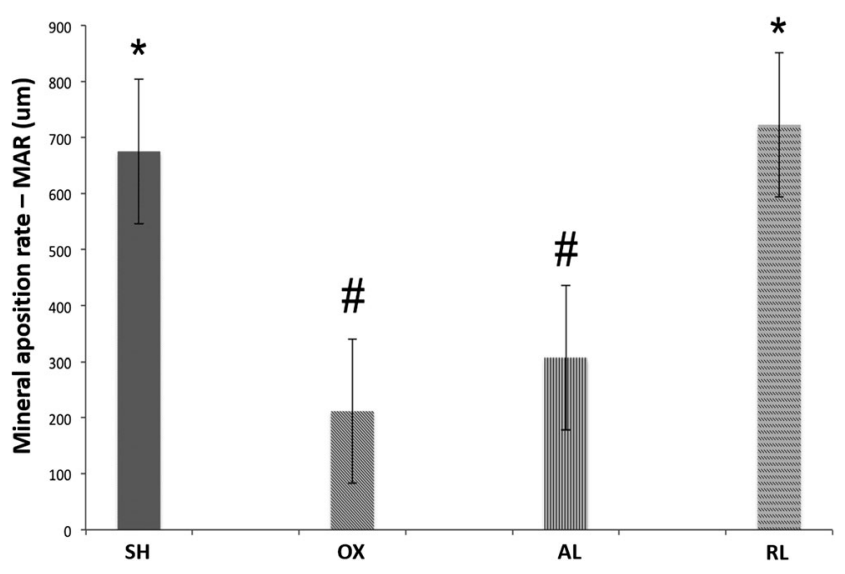

Fig. 5 Peri-implant bone area $\left(\mu \mathrm{m}^{2}\right)$ of osteoporosis groups ( $\mathrm{SH}, \mathrm{OX}$, $\mathrm{AL}$, and RL) according to calcein and alizarin staining $(P<0.05$, comparisons among the groups showing statistical significance)

A discrete positive staining was observed for OPG in the extracellular matrix of bone tissue, as this protein is classified as a soluble receptor and, after its synthesis by osteoblasts, it traverses the plasma membrane into the extracellular environment (Figs. 7 and 8) [19].

Osteocalcin is the main noncollagen protein of the extracellular matrix of bone tissue, characterizing bone tissue mineralization, as well as its stage of maturity. In the study groups, osteocalcin revealed a moderate score (2) at 42 days in the $\mathrm{SH}$ group. In the OX group, staining was also moderate (2). In the AE group, it showed moderate score (2) at 42 days.

Raloxifene showed an inverse response pattern, presenting moderate staining at 42 days. Osteocalcin was present in cells of the osteoblastic lineage and in the precipitate in the extracellular bone matrix. TRAP is an enzyme that indicates osteoclast resorption activity in the bone tissue. After 42 days, TRAP staining was moderate (2) in the $\mathrm{SH}$ group and intense in the OX group (3).

In the AL group, a light staining was observed (1), and in the RL group, like the SH group, moderate staining was present (2).

At 42 days, OPG protein showed higher labeling for the $\mathrm{AL}$ and RL groups in both periods $(P<0.05$, Tukey test). Over time, the RL group showed an increase in osteocalcin labeling, while the AL group showed a decrease for this protein $(P<0.001$, Tukey test). The TRAP protein presented the highest labeling for $\mathrm{OX}$, followed by the RL, SH, and $\mathrm{AL}$ groups $(P>0.05$, Dunn's test).

\section{Micro-CT analysis}

Inconsistent with the other measures, the micro-CT of the narrow region of interest showed that alendronate supports bone formation, as indicated by percentage bone volume and trabecular number, compared to the $\mathrm{OX}$ alone group and was even higher than the raloxifene group. Consequently, microCT analysis does not necessarily represent the conditions immediately adjacent to the implants, where no advantage with alendronate was observed. The percentage of bone volume (BV/TV) presented better results in the treated groups (AL and RL), with the greatest values in group AL, followed by groups RL, OX, and SH (Fig. 9a).

The trabecular thickness did not show any evidence of significant changes $(P=0.953$, Kruskal-Wallis), although the results in the group treated with raloxifene $(0.05 \pm 0.007 \mathrm{~mm})$ were closer to the $\mathrm{SH}$ group $(0.051 \pm 0.009 \mathrm{~mm})$. Lower values were observed in the alendronate group $(0.04 \pm 0.007 \mathrm{~mm})$.

Individual evaluation of the experimental groups for trabecular thickness and number and separation of trabeculae showed that the trabecular thickness was lower in group AL, confirmed by the greater number of trabeculae (Tr.N; Fig. 9b) and the lower separation between them (Tr.S; Fig. 9c). In the osteoporotic animals (OX), lower Tr.Th, lower Tr.N, and greater Tr.S were observed. In the evaluation between the groups, the comparisons $\mathrm{SH} \times \mathrm{AL}$ and $\mathrm{OX} \times \mathrm{AL}$ presented statistical significance $(P<0.05$, Holm-Sídak) for Tr.N, as did the comparison $\mathrm{OX} \times \mathrm{AL}$ for the parameter $\operatorname{Tr} . \mathrm{S}(P=0.08$, Holm-Sídak).

\section{Molecular analysis (PCR)}

The most significant finding was that the ratio of RANKL to OPG was rather similar among the $\mathrm{SH}$ group and the $\mathrm{OX}$
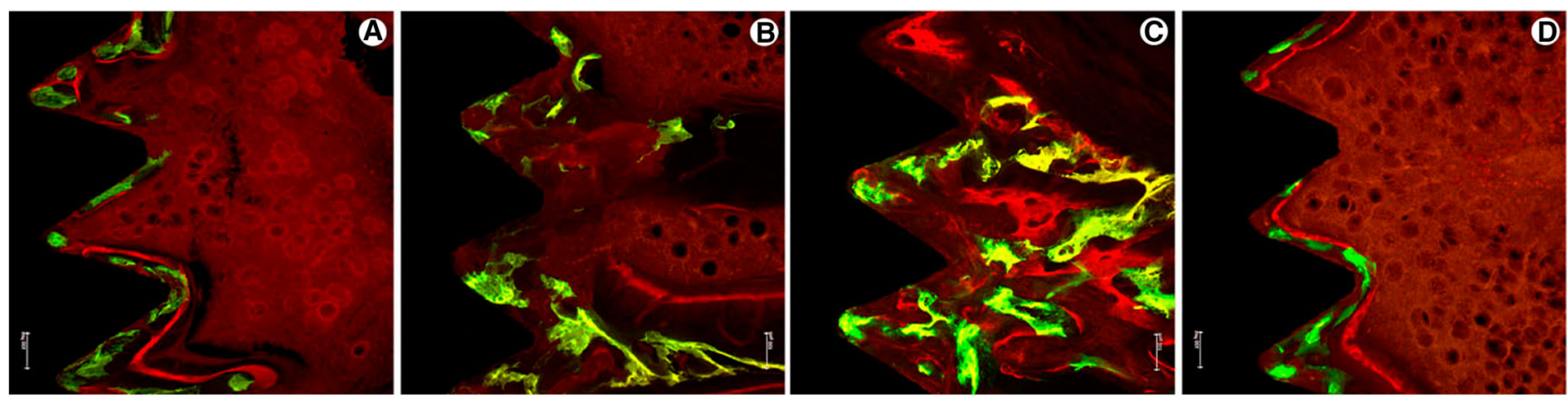

Fig. 6 Mineral aposition rate (MAR, $\mu \mathrm{m})$ calculated through fluorochrome images (beginning of calcein precipitation up to alizarin red precipitation) and divided per 28 days (interval of analysis). *, \# denotes statistically significance difference $(P<0.05)$ 


\section{OPG}
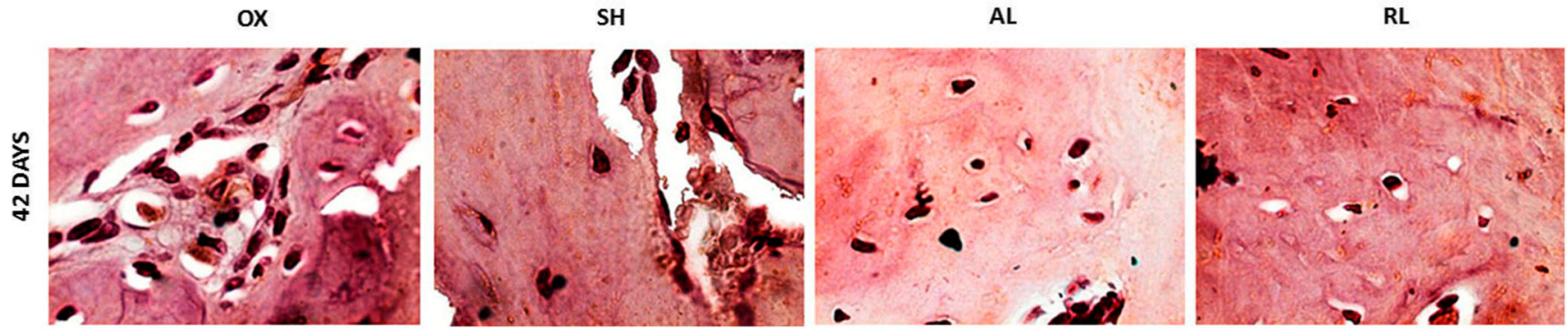

Fig. 7 Immunostaining of osteoprotegerin (OPG) at 42 days in osteoporosis group ( $\mathrm{SH}, \mathrm{OX}, \mathrm{AL}$, and $\mathrm{RL}$ ). The red arrows indicate staining areas-1 mild; 2 moderate; 3 intense. 42 days: OX (1), SH (1), AL (2), and RL (2); original magnification: $\times 40$

group alone and the raloxifene group, while alendronate caused a substantial inverse of RANKL over OPG expression (Fig. 10). Therefore, alendronate leads to a decrease in bone resorption and to a compensatory increase of the RANKL-toOPG ration in the sense of a feed-back loop.

\section{Discussion}

The main finding of the present study was that raloxifene but not alendronate supported osseointegration of titanium implants placed in the tibia of OX rats. These findings are supported by the traditional parameters of bone-to-implant contact and bone volume. Also in support of this suggestion was the similar pattern of fluorochrome labeling in the OX-alone and the alendronate group - with raloxifene having a similar pattern in the SH control group. The micro-CT data, however, were not consistent with the two-dimensional measures, as alendronate supports the percentage bone volume and trabecular number, and in the gene expression analysis, alendronate increased RANKL over OPG expression. In the micro-CT results, the only difference observed was found in number and separation of trabeculae. The other parameters did not show a difference, but a tendency that AL is better with regard to bone volume and raloxifene, and similarly $\mathrm{SH}$, is better in trabecular thickness. So, AL has a tendency to give good results, but not much different than raloxifene. This $\mathrm{AL}$ response could be attributed to the blocked osteoclast activity, which could result in a greater bone volume and number of bone trabeculae. The other experiments also show the superiority of raloxifene. These findings are important as they show that the final conclusion of whether osteoporosis therapies support osseointegration can depend on the method of evaluation.

There is interesting data about peri-implant bone healing and bone dynamics in the area of calcein precipitation for the $\mathrm{AL}$ group. Bone turnover (calcein/alizarin) was lower when compared with the RL and SH groups. On the other hand, the RANKL/OPG ratio through relative gene expression showed higher results for AL than RL, whereas micro-CT parameters showed a higher bone volume for AL. This seems controversial, but it can be explained by the action mechanism of bisphosphonate. The anti-resorptive action is produced by binding to hydroxyapatite due to the pyrophosphate analog effect, which inhibits osteoclast function and promotes apoptosis of the osteoclasts [6, 28]. Furthermore, all organism metabolic reactions are dependent on negative feedback. Thus, decreasing osteoclastic action caused by alendronate will promote an increase of RANKL precipitation. However, osteoclasts presented lower function. There was no active resorption and a large amount of old bone without bone turnover, verified by greater calcein precipitation in the AL group.

Clinically, the major concern regards long-term impact of $\mathrm{AL}$ administration and oscillations in the oral cavity

\section{RANKL}
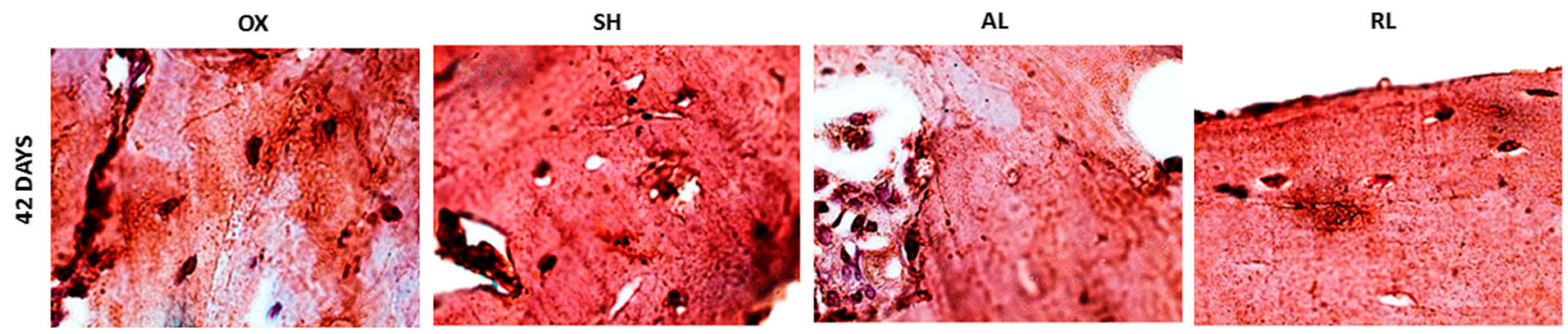

Fig. 8 Immunostaining of RANKL to 42 days in the osteoporosis group ( $\mathrm{SH}, \mathrm{OX}, \mathrm{AL}$, and RL). The red arrows indicate areas of staining-1 mild; 2 moderate; 3 intense. 42 days: $\mathrm{OX}(2), \mathrm{SH}(2), \mathrm{AL}(2)$, and RL (2); original magnification: $\times 40$ 


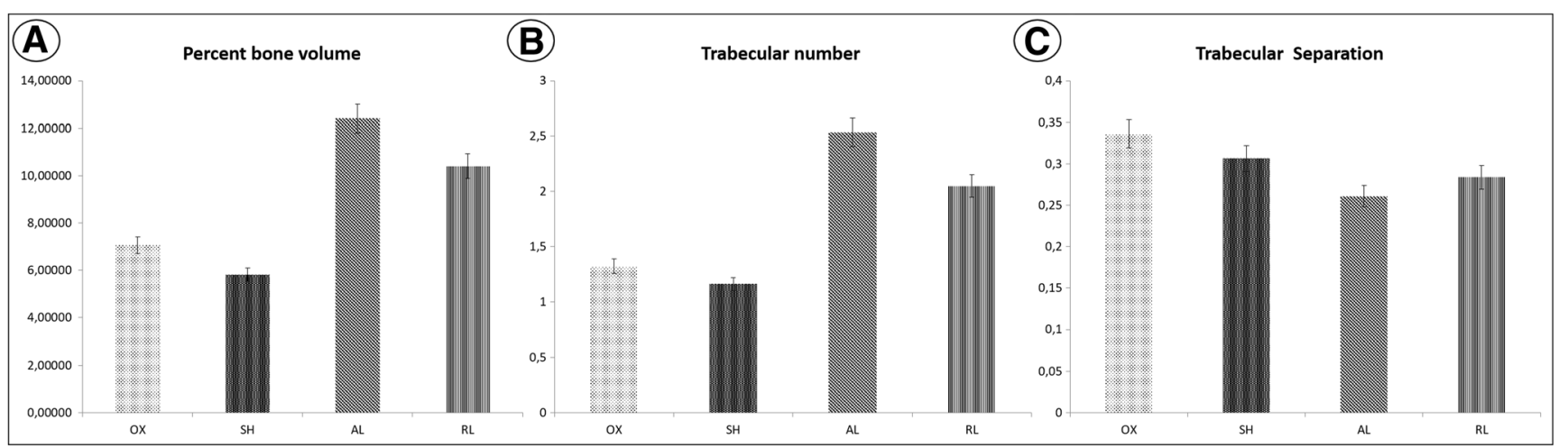

Fig. 9 a Average and standard deviation of bone volume percentage (BV/TV) obtained in microtomography analysis for the OX, SH, AL, and RL at 42 days. There was no statistically significant difference in comparisons among experimental groups $(P=0.729$, one-way ANOVA). $\mathbf{b}$ Average and standard deviation of the number of trabecular (Tr.N) obtained in microtomographic analysis for OX, SH, AL, and RL at

42 days. The $\mathrm{SH} \times \mathrm{AL}$ and $\mathrm{OX} \times \mathrm{AL}$ comparisons showed statistically significant differences $(P<0.05$, Holm-Sídak). c Average and standard deviation of the separation of trabeculae (Tr.S) obtained in microtomographic analysis for $\mathrm{OX}, \mathrm{SH}, \mathrm{AL}$, and $\mathrm{RL}$ at 42 days. The highest value was for the $\mathrm{OX}$ group and the lowest value for group $\mathrm{AL}$ $(\mathrm{OX} \times \mathrm{AL}, P=0.008$, Holm-Sídak $)$

throughout life. It seems AL promotes a freezing in the bone tissue. Thus, throughout life, peri-implant bone will be submitted to microtraumas that, with an anti-resorptive drug like alendronate, can cause osteonecrosis.

The findings are therefore only partially conclusive with the findings of a review integrating similar research in 12 studies showing that systemic bisphosphonates significantly increased bone volume and bone-to-implant contact under osteoporotic conditions. But also in this context, two studies failed to find a significant difference in osseointegration among OX animals [18]. For example, zoledronic acid caused a negligible change in bone-toimplant contacts and bone area in OX and tibia $[15,16$, 29]. There was also a higher removal torque by alendronate in OX rat tibias, but bone-to-implant contacts showed no considerable changes [13], which is in support of the present study, in which micro-CT data show a beneficial effect of alendronate on osseointegration. Also, our recent study revealed a moderately higher removal torque with alendronate and particularly with raloxifene [10].

The clinical relevance of the present findings has to be interpreted in the context of similar preclinical studies but also in the context of dental implants placed in osteoporotic patients, many of which receive anti-resorptive therapies or raloxifene. Obviously, alendronate does not hinder or impair the process of osseointegration, thus allowing new peri-implant bone formation. However, whether the functional adaptation, which is based in bone remodeling, affects clinical success remains an open question. Observations from this study where fluorochrome labeling was used to show peri-implant bone remodeling even suggest that alendronate moderately supports bone turnover, indicated by calcein and alizarin red incorporation during osseointegration. More consistent are the experimental data with raloxifene [10]; however, any clinical conclusions require more research.

The study has limitations. First, the study is underpowered because of the multiple end points that were included in the analysis. Nevertheless, the data provide some preliminary direction and should be interpreted in the context of the existing

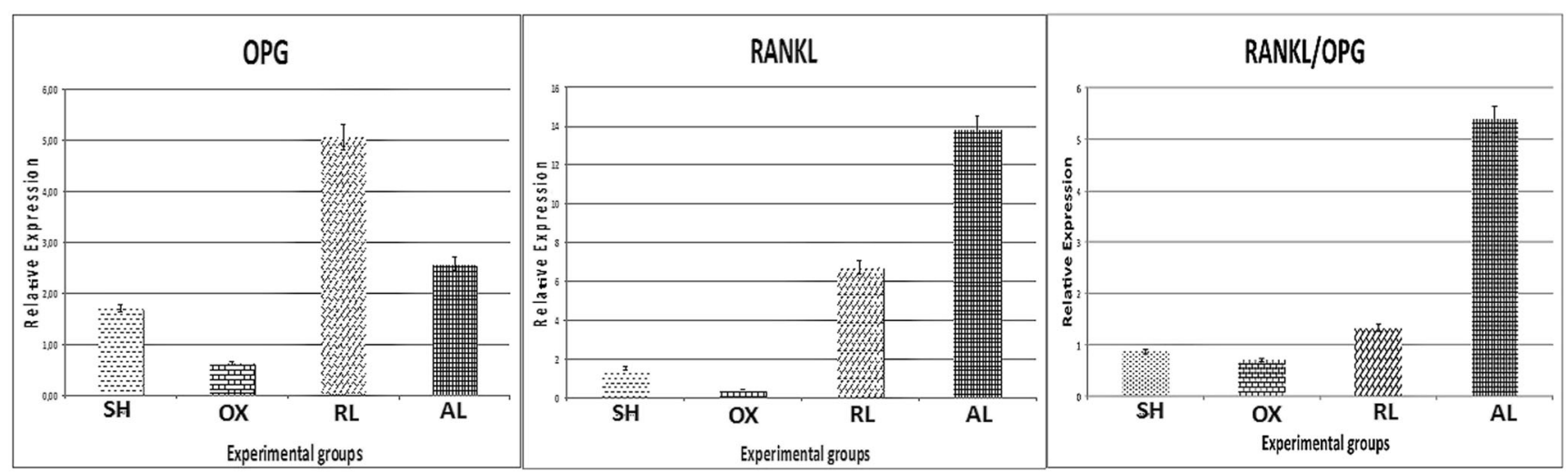

Fig. 10. Relative gene expression of OPG, RANKL, and RANKL/OPG ratio by real-time PCR analysis of the experimental groups (OX, SH, AL, and $\mathrm{RL}) . P<0.05$ denotes statistical significance differences $(\mathrm{SH} \times \mathrm{RL})$ and $(\mathrm{AL} \times \mathrm{RL})$. 
knowledge, which is significant for alendronate and other bisphosphonates but limited for raloxifene. Second, the rat tibia model does not necessarily reflect the oral situation where implants are subjected to functional loading. Thus, the model only represents the healing phase and not the long-term functional status of implants in osteoporotic patients. Finally, the study remains descriptive, as the molecular and cellular mechanisms that lead to the changes in osseointegration, particularly with raloxifene, remain unknown, even though we have some preliminary data on the included RANKL/OPG ratio. The present data at least support future attempts to understand how raloxifene significantly supports osseointegration.

\section{Conclusions}

Raloxifene turned out to be of possible benefit in stimulating osseointegration, at least in osteoporotic rats, while the impact of alendronate to support the process was comparably weak and not obviously different from the OX controls. It is thus raloxifene and not alendronate that counteracts diminished osseointegration in osteoporotic rats.

Acknowledgments The authors would like to thank the São Paulo Research Foundation-FAPESP (2012/15748-8 and 2012/15912-2), for financial support; Marcia Sirlene Zardin Graeff, from Bauru Dental School-USP, for help in obtaining confocal microscopy images, and Prof. Dr. Elcio Marcantonio Junior and laboratory technician Ana Claudia Gregolin Costa Miranda, from UNESP-Araraquara, for facilitating the laboratory processing of specimens with EXAKT. Also, we would like to thank the Implalife Biotechnology for providing the implants.

\section{Compliance with ethical standards}

Conflict of interest The authors declare that they have no conflict of interest.

Funding This study was supported by the São Paulo Research Foundation-FAPESP (2012/15748-8 and 2012/15912-2).

Ethical approval The procedures and protocol design described here were approved by the Ethics Committee in Animal Experimentation of Aracatuba Dental School, UNESP — Univ Estadual Paulista, Brazil (approval no. 2012-01096).

Informed consent For this type of study, formal consent is not required.

\section{References}

1. Yoshimura N et al (2009) Prevalence of knee osteoarthritis, lumbar spondylosis, and osteoporosis in Japanese men and women: the research on osteoarthritis/osteoporosis against disability study. J Bone Miner Metab 27(5):620-628

2. Tanaka S et al (2016) Real-world evidence of raloxifene versus alendronate in preventing non-vertebral fractures in Japanese women with osteoporosis: retrospective analysis of a hospital claims database. J Bone Miner Metab. doi:10. 1007/s00774-016-0809-0

3. Chen $\mathrm{H}$ et al (2013) Smoking, radiotherapy, diabetes and osteoporosis as risk factors for dental implant failure: a meta-analysis. PLoS One 8(8):e71955

4. Busenlechner D et al (2014) Long-term implant success at the academy for oral implantology: 8-year follow-up and risk factor analysis. J Periodontal Implant Sci 44(3):102-108

5. Khan M, Cheung AM, Khan AA (2017) Drug-related adverse events of osteoporosis therapy. Endocrinol Metab Clin N Am 46(1):181-192

6. Giro $\mathrm{G}$ et al (2011) The effect of oestrogen and alendronate therapies on postmenopausal bone loss around osseointegrated titanium implants. Clin Oral Implants Res 22(3):259-264

7. Tsurushima $\mathrm{H}$ et al (2013) Bacterial promotion of bisphosphonateinduced osteonecrosis in Wistar rats. Int J Oral Maxillofac Surg 42(11):1481-1487

8. Gossiel F et al (2016) The effect of bisphosphonate treatment on osteoclast precursor cells in postmenopausal osteoporosis: the TRIO study. Bone 92:94-99

9. Russell RG (2011) Bisphosphonates: the first 40 years. Bone 49(1): 2-19

10. Ramalho-Ferreira $\mathrm{G}$ et al (2015) Raloxifene enhances peri-implant bone healing in osteoporotic rats. Int J Oral Maxillofac Surg 44(6): 798-805

11. Lama A et al (2017) Extracorporeal shock waves alone or combined with raloxifene promote bone formation and suppress resorption in ovariectomized rats. PLoS One 12(2):e0171276

12. Urano T et al (2017) Preventive effects of raloxifene treatment on agerelated weight loss in postmenopausal women. J Bone Miner Metab 35(1):108-113

13. Narai S, Nagahata S (2003) Effects of alendronate on the removal torque of implants in rats with induced osteoporosis. Int J Oral Maxillofac Implants 18(2):218-223

14. Skripitz R et al (2009) Effect of alendronate and intermittent parathyroid hormone on implant fixation in ovariectomized rats. J Orthop Sci 14(2):138-143

15. Dikicier E et al (2014) Effect of systemic administered zoledronic acid on osseointegration of a titanium implant in ovariectomized rats. J Craniomaxillofac Surg 42(7):1106-1111

16. Chen B et al (2013) Zoledronic acid enhances bone-implant osseointegration more than alendronate and strontium ranelate in ovariectomized rats. Osteoporos Int 24(7):2115-2121

17. de Oliveira MA et al (2015) The effects of zoledronic acid and dexamethasone on osseointegration of endosseous implants: histological and histomorphometrical evaluation in rats. Clin Oral Implants Res 26(4):e17-e21

18. Vohra F et al (2014) Efficacy of systemic bisphosphonate delivery on osseointegration of implants under osteoporotic conditions: lessons from animal studies. Arch Oral Biol 59(9):912-920

19. Evans HM, Long JA (1922) Characteristic effects upon growth, oestrus and ovulation induced by the Intraperitoneal Administration of Fresh Anterior Hypophyseal Substance. Proc Natl Acad Sci U S A 8(3):38-39

20. Teofilo JM et al (2003) Comparison between two experimental protocols to promote osteoporosis in the maxilla and proximal tibia of female rats. In Pesqui Odontol Bras Brazil p 302-6 
21. Ramalho-Ferreira G et al (2015) Alveolar bone dynamics in osteoporotic rats treated with raloxifene or alendronate: confocal microscopy analysis. J Biomed Optics 20(3)

22. da Paz LH et al (2001) Effect of 17beta-estradiol or alendronate on the bone densitometry, bone histomorphometry and bone metabolism of ovariectomized rats. In Braz J Med Biol Res Brazil p 1015-22

23. Luvizuto ER et al (2010) Histomorphometric analysis and immunolocalization of RANKL and OPG during the alveolar healing process in female ovariectomized rats treated with oestrogen or raloxifene. In Arch Oral Biol 2009 Elsevier Ltd: England p 52-9

24. Luvizuto ER et al (2010) Osteocalcin immunolabeling during the alveolar healing process in ovariectomized rats treated with estrogen or raloxifene. In Bone 2009 Elsevier Inc: United States p 1021-9

25. Ramalho-Ferreira G et al (2015) Alveolar bone dynamics in osteoporotic rats treated with raloxifene or alendronate: confocal microscopy analysis. J Biomed Opt p 038003-1 - 038003-7
26. Dempster DW et al (2013) Standardized nomenclature, symbols, and units for bone histomorphometry: a 2012 update of the report of the ASBMR Histomorphometry Nomenclature Committee. J Bone Miner Res 28(1):2-17

27. Blackledge NP, Rose NR, Klose RJ (2015) Targeting polycomb systems to regulate gene expression: modifications to a complex story. Nat Rev Mol Cell Biol 16(11):643-649

28. Giro G et al (2011) Influence of estrogen deficiency on bone around osseointegrated dental implants: an experimental study in the rat jaw model. J Oral Maxillofac Surg 69(7):1911-1918

29. Cardemil C et al (2013) The effects of a systemic single dose of zoledronic acid on post-implantation bone remodelling and inflammation in an ovariectomised rat model. Biomaterials 34(5):15461561 